\title{
Guest editorial: cancer immunology
}

\author{
Masaki Yasukawa
}

Received: 9 February 2011/Revised: 14 February 2011/ Accepted: 14 February 2011/Published online: 25 February 2011

(c) The Japanese Society of Hematology 2011

Recent progress in cancer chemotherapy has improved the outcome of hematological malignancies, but cure rates remain unsatisfactory and treatments are often associated with severe adverse events. The search for new strategies based on novel concepts is therefore intense. Recent data from in vivo experiments using animal models and clinical observations of patients with leukemia who have undergone allogeneic hematopoietic stem cell transplantation (HSCT) have shown that immune surveillance plays an important role in the achievement of cure. Patients with leukemia who undergo T cell-depleted HSCT appear to have a significantly higher relapse rate than those who undergo conventional HSCT. It has also been reported that the relapse rate in leukemia patients who undergo HSCT with a graft from an identical twin is higher than that in patients who receive a graft from a non-identical sibling. These clinical observations strongly suggest that the inhibitory effect of cancer chemotherapy on tumor cell growth is limited, and that an immune response against malignant cells mediated by immune-competent cells, $\mathrm{T}$ lymphocytes in particular, is essential for achieving cure of malignancies. This concept has inspired a race to develop cell-mediated immunotherapy targeting tumor-associated antigens.

The development of safe and effective cancer immunotherapies will require the identification of tumor-associated antigens that can be recognized by human CD4+

M. Yasukawa ( $\square)$

Department of Bioregulatory Medicine, Ehime University Graduate School of Medicine, Toon, Ehime 791-0295, Japan

e-mail: yasukawa@m.ehime-u.ac.jp

M. Yasukawa

Proteo-Medicine Research Center, Ehime University,

Toon, Ehime 791-0295, Japan and CD8+ T lymphocytes. Since the cloning of the gene encoding melanoma-associated antigen, MAGE-1, in 1991, various types of human tumor-associated antigens that are recognized by human $\mathrm{T}$ lymphocytes have been identified. Promising data from basic and preclinical studies have prompted a number of clinical trials of cell-mediated immunotherapy for malignancies. These include vaccination with peptides derived from tumor-associated antigens, vaccination with dendritic cells, adoptive transfer of tumorreactive $\mathrm{T}$ cells, and gene-engineered $\mathrm{T}$ cell therapy. Although cell-mediated immunotherapy for cancer by targeting tumor-associated antigens appears to be a promising strategy, the clinical responses obtained in these recent trials have not been satisfactory.

Tumors evade immune surveillance in many ways. Recent studies have revealed that various types of immune cell, including regulatory $\mathrm{T}$ cells, myeloid-derived suppressor cells, and regulatory dendritic cells, negatively regulate the anti-tumor immune response in vivo. It has also been revealed that cancer cells build an immunosuppressive microenvironment through a variety of immunosuppressive factors. Negative immunological regulation must therefore be overcome in order to establish optimal tumor immunotherapy. Although data from recent fundamental studies of cancer immunology and molecular oncology have been translated into clinical applications, many questions are yet to be answered. What is the most efficient target antigen for $\mathrm{T}$ cell-mediated immunity against cancers? What is the most effective vaccination adjuvant for inducing an anti-tumor immune response? How should immunization with tumor-associated antigens be conducted in order to induce an effective anti-tumor immune response? What is the best approach for the accurate evaluation of the clinical efficacy of a cancer immunotherapy? Further studies are needed to answer 
these important questions, and to establish safe and efficacious cancer immunotherapies.

In Progress in Hematology in this issue, several experts in tumor immunology give an overview of recent progress in this field. Dr. Robert C. Rees and colleagues review the tumor-associated antigens identified to date, and consider their potential for use in tumor immunotherapy. Dr. Katayoun Rezvani and colleagues survey ongoing clinical trials of peptide vaccine therapy for leukemia, and discuss future prospects. Dr. Antonio Rosato and colleagues discuss immunotherapy for Epstein-Barr virus-associated malignancies with an eye to clinical applications of virus-specific $\mathrm{T}$ cell therapy for cancers. Finally, Dr. Yutaka Kawakami and colleagues look at the mechanisms by which cancers evade immune surveillance, and the development of strategies to overcome this problem. I believe these reviews will be of interest to readers seeking to gain insights into the current status and future prospects of curative immunotherapy for cancers. 